\title{
ON ELEMENTS WITH NEGATIVE SQUARES
}

\author{
RALPH DEMARR AND ARTHUR STEGER
}

\begin{abstract}
We prove that in a partially ordered linear algebra no element can have a square which is the negative of an order unit. In particular, the square of a real matrix cannot consist entirely of negative entries. We generalize the well-known theorem that the complex numbers admit no lattice order.
\end{abstract}

It is a simple matter, using the Perron-Frobenius theorem [3], to show that the square of a real (finite) matrix cannot consist entirely of negative entries. In this paper we give an alternate proof of this result which makes use only of some elementary order properties. It is appropriate, therefore, to construct a proof within the more general framework of partially ordered linear algebras. The main theorem will then be valid not only for finite matrices but also for (row-finite) infinite matrices as well as operators on a Banach space [1]. We also generalize a result due to Birkhoff and Pierce [2] which states that the complex number field regarded as a linear algebra over the reals admits no lattice order.

Definition. A partially ordered linear algebra (p.o.l.a.) $P$ is a real associative linear algebra on which there is defined a partial ordering which satisfies the following conditions $(x, y, z$ denote elements of $P$ and $\alpha$ denotes a real number):

(a) if $x \leqq y$ then $x+z \leqq y+z$,

(b) if $0 \leqq x$ and $0 \leqq y$ then $0 \leqq x y$,

(c) if $0 \leqq \alpha$ and $0 \leqq x$ then $0 \leqq \alpha x$,

(d) for any $x$ there exist $y \geqq 0$ and $z \geqq 0$ such that $x=y-z$. We also make use of the following condition:

$\left(d^{*}\right)$ for any $x$ there exists a $y$ such that $-y \leqq x \leqq y$.

It is easy to verify that conditions (a), (b), (c), and (d) are equivalent to (a), (b), (c), and ( $\left.\mathrm{d}^{*}\right)$. It can be shown, however, that a stronger set of conditions is obtained if (d) is replaced by the condition that the partial ordering be a lattice order. We make use of the standard elementary

Received by the editors February 17, 1971.

AMS 1970 subject classifications. Primary 06A70; Secondary 15A45, 47B55.

Key words and phrases. Partially ordered linear algebras, matrix inequalities, linear operators. 
arithmetic properties which flow directly from (a), (b), (c), and (d). We note explicitly, however, the following:

Lemma 1. Let $P$ be a p.o.l.a., let $x, y, u, v \in P$ and let $\alpha, \beta$ be real numbers.

(1) If $-u \leqq x \leqq u$ and $-v \leqq y \leqq v$, then $-u v \leqq x y \leqq u v$.

(2) If $-\alpha \leqq \beta \leqq \alpha$ and $-u \leqq x \leqq u$, then $-\alpha u \leqq \beta x \leqq \alpha u$.

Proof. We give only the proof of (1). From $0 \leqq u-x$ and $0 \leqq$ $v+y$, it follows that $0 \leqq u v-x y+u y-x v$. On the other hand, from $0 \leqq u+x$ and $0 \leqq v-\bar{y}$, we obtain $0 \leqq u v-x y-u y+x v$. Therefore, $0 \leqq 2 u v-2 x y, 0 \leqq u v-x y$, and $x y \leqq u v$. Similarly, from $-u \leqq$ $-x \leqq u$ and $-v \leqq y \leqq v$, we obtain $-x y \leqq u v$. Thus, $-u v \leqq x y \leqq u v$.

Lemma 2. Let $P$ be a p.o.l.a. and let $x, u \in P$. If $0 \leqq u, x \leqq u, x^{2} \leqq 0$, and $x u=u x$, then $n u^{n-1} x \leqq u^{n}$ for $n \geqq 1$.

Proof. We proceed by induction and observe that the case $n=1$ is included in the hypothesis. The proof is completed simply by multiplying $0 \leqq u^{n}-n u^{n-1} x$ by $0 \leqq u-x$ to obtain

$$
0 \leqq u^{n+1}-(n+1) u^{n} x+n u^{n-1} x^{2} \leqq u^{n+1}-(n+1) u^{n} x .
$$

If $P$ is a p.o.l.a. and $B$ is a subalgebra of $P$, the nonnegative element $u \in B$ is said to be an order unit for $B$ provided that for every $x \in B$ there exists a real number $\gamma$ such that $-\gamma u \leqq x \leqq \gamma u$. If $y \in P$ then the subalgebra of $P$ generated by $y$ is denoted by $A(y)$.

MAIn Theorem. Let $P$ be a p.o.l.a., let $x \in P$, and let $x^{2}=-y \leqq 0$. If there is an element $w \in A(y)$ such that (i) $w$ is an order unit for $A(y)$ and (ii) $-w \leqq x \leqq w$, then $x=0$.

Proof. Since $w$ is an order unit for $A(y)$, there is a real number $\beta \geqq 1$ such that $y \leqq \beta w$. Define $u=\beta w$. Then $u=\sum_{k=1}^{t} \alpha_{k} y^{k}$ for some positive integer $t$ and for some real numbers $\alpha_{1}, \alpha_{2}, \cdots, \alpha_{t}, u$ is an order unit for $A(y),-u \leqq x \leqq u$, and $-u \leqq y \leqq u$. By Lemma 2 , we conclude that $n u^{n-1} x \leqq u^{n}$ and $n u^{n-1}(-x) \leqq u^{n}$ for $n \geqq 1$. Thus, $-u^{n} \leqq n u^{n-1} x \leqq u^{n}$ and, multiplying by $-u \leqq-x \leqq u$, we obtain $n u^{n-1} y \leqq u^{n+1}$ for $n \geqq 1$. We note, for later reference, that $n=1$ yields $y \leqq u^{2}$. From $u^{3}=$ $\left(\sum_{k=1}^{t} \alpha_{k} y^{k}\right)^{3}$, we have $u^{3}=y z$ for some $z \in A(y)$. If we choose $\delta$ so that $z \leqq \delta u$, we have $u^{3} \leqq \delta u y$. Hence, $n u^{n+1} \leqq \delta n u^{n-1} y \leqq \delta u^{n+1}$ for $n \geqq 2$. If $N$ is chosen so that $N>\delta$, we can conclude that $u^{N+1}=0$. From $0 \leqq y \leqq u$ and $0 \leqq y \leqq u^{2}$, we obtain $0 \leqq y^{n} \leqq u^{n+1}$ for $n \geqq 1$. Therefore $y^{N}=0$ and, since $u \in A(y), u^{N}=0$. Thus, $y^{N-1}=0, u^{N-1}=0$, etc. back to $u=0$. Finally, $-u \leqq x \leqq u$ implies that $x=0$.

The algebra $R_{n}$ of real $n \times n$ matrices can be partially ordered in infinitely many ways so as to produce a p.o.l.a. In this paper, however, we consider just one such ordering; namely, if $x=\left(\alpha_{i j}\right)$ and $y=\left(\beta_{i j}\right)$ are 
matrices in $R_{n}$, we say that $x \leqq y$ provided $\alpha_{i j} \leqq \beta_{i j}$ for $1 \leqq i, j \leqq n$. It is immediate that this partial ordering of $R_{n}$ does indeed yield a p.o.l.a. Further, we say that the matrix $x=\left(\alpha_{i j}\right)$ is positive provided $\alpha_{i j}>0$ for $1 \leqq i, j \leqq n$.

COROLlaRY 1. Let $R_{n}$ be the p.o.l.a. of real $n \times n$ matrices and let $x \in R_{n}$. If $x^{2}=-y \leqq 0$, then there are no positive matrices in $A(y)$.

Proof. Suppose the corollary is false and let $v$ be a positive matrix in $A(y)$. Then $v$ is clearly an order unit for $A(y)$ and, in addition, we may choose a real number $\gamma$ such that $-\gamma v \leqq x \leqq \gamma v$. The Main Theorem guarantees that $x=0$. Thus, $y=0$ and $v=0$, a contradiction.

The following examples are of interest.

EXAMPLE 1. Let $y \in R_{n}$ where

$$
y=\left[\begin{array}{ccccc}
0 & 1 & 0 & \cdots & 0 \\
0 & 0 & 1 & \cdots & 0 \\
\cdot & & & & \\
\cdot & & & & \\
\cdot & & & & \\
0 & 0 & 0 & \cdots & 1 \\
1 & 0 & 0 & \cdots & 0
\end{array}\right] .
$$

It is easy to verify that $y+y^{2}+\cdots+y^{n}$ is a positive matrix. Hence $x^{2} \neq-y$ for every $x \in R_{n}$.

EXAMPLE 2. Let $P$ be the algebra of all (row-finite) infinite matrices. If $x=\left(\alpha_{i j}\right)$ and $y=\left(\beta_{i j}\right)$ are in $P$, we say that $x \leqq y$ provided $\alpha_{i j} \leqq \beta_{i j}$ for all $i, j$. Under this partial ordering $P$ becomes a p.o.l.a. Let $x=$ $\left(\gamma_{i j}\right) \in P$ where

$$
\begin{aligned}
\gamma_{i j} & =(-1)^{i} i & \text { if } & 2 \leqq j \leqq i+1,
\end{aligned} \quad \text { for } i=1,2, \cdots .
$$

If $y=-x^{2}$, it is easily seen that $-y \leqq x \leqq y$. This example shows that in the Main Theorem (ii) alone is not enough. Clearly, (i) alone is not enough; simply take $x \neq 0$ and $x^{2}=0$.

EXAMPLE 3. Let $y \in R_{n}$ with $0 \leqq y$. It is easy to show that if $y$ has at most $n-2$ zero entries then $y^{2}$ is a positive matrix. Thus, by Corollary 1 , if $x^{2}=-y \leqq 0$, then $y$ has at least $n-1$ zero entries. There is some evidence to suggest that, if $x \in R_{n}(n \geqq 2)$ and $x^{2}=-y \leqq 0$, then there is a permutation matrix $p$ such that

$$
p^{-1} y p=\left[\begin{array}{cc}
y_{11} & 0 \\
0 & y_{22}
\end{array}\right]
$$

where $y_{11}$ and $y_{22}$ are square matrices of order less than $n$. If this were true it would follow that $y$ has at least $2 n-2$ zero entries. This latter 
result cannot be improved upon; that is, for every $n \geqq 2$ there is a matrix $x \in R_{n}$ such that $x^{2} \leqq 0$ and $x^{2}$ has exactly $2 n-2$ zeros. For example: Let

$$
x=\left[\begin{array}{ccccc}
-1 & 2 & 2 & \cdots & 2 \\
-2 & & & & \\
-2 & & & & \\
\cdot & & & I_{22} & \\
\cdot & & & & \\
\cdot & & & & \\
-2 & & & &
\end{array}\right]
$$

where $I_{22}$ is the identity matrix of order $n-1$. If $y=-x^{2}$, then

$$
y=\left[\begin{array}{ccccc}
y_{11} & 0 & 0 & \cdots & 0 \\
0 & & & & \\
0 & & & & \\
\cdot & & & y_{22} & \\
\cdot & & & & \\
\cdot & & & & \\
0 & & & &
\end{array}\right]
$$

where $y_{11}$ and $y_{22}$ are positive matrices of order 1 and $n-1$, respectively.

COROLlaRY 2. Let A be a finite dimensional real linear algebra with $1 \neq 0$. If there is an element $i$ in the center of $A$ such that $i^{2}=-1$, then $A$ cannot be partially ordered so that $A$ becomes a p.o.l.a.

Proof. Assume $A$ is a p.o.l.a. under some partial ordering and let $\left\{w_{1}, w_{2}, \cdots, w_{n}\right\}$ be a basis for $A$. Choose $u_{k}$ so that $-u_{k} \leqq w_{k} \leqq u_{k}$ for $1 \leqq k \leqq n$ and put $u=\sum_{k=1}^{n} u_{k}$. Now, suppose $w=\sum_{i=1}^{n} \gamma_{i} w_{i} \in A$ and let $\gamma=\max \left\{\left|\gamma_{1}\right|,\left|\gamma_{2}\right|, \cdots,\left|\gamma_{n}\right|\right\}$. Then $-\gamma u_{k} \leqq \gamma_{k} w_{k} \leqq \gamma u_{k}$ for $1 \leqq k \leqq n$ and $-\gamma u \leqq w \leqq \gamma u$. Thus, $u$ is an order unit for $A$. Hence, for some real number $\beta,-\beta u \leqq 1 \leqq \beta u$. If $v=\beta u$, then $1 \leqq v$ implies that $v \leqq v^{2}$. Therefore, $v^{2}$ is an order unit for $A$. Now let $x=i v$. Then $x^{2}=(i v)^{2}=$ $-v^{2}$. By the Main Theorem, $x=0$. Thus $v^{2}=0$ and $1=0$, a contradiction.

\section{REFERENCES}

1. Ralph DeMarr, On partially ordering operator algebras, Canad. J. Math. 19 (1967), 636-643. MR 35 \#3450.

2. L. Fuchs, Partially ordered algebraic systems, Pergamon Press, New York, 1963, p. 143. MR 30 \#2090.

3. P. Lancaster, Theory of matrices, Academic Press, New York, 1969, pp. 282-291. MR 39 \#6885

Department of Mathematics, University of New Mexico, Albuquerque, New MeXICo 87106 